\title{
BMJ Open Use of tabletop exercises for healthcare education: a scoping review protocol
}

\author{
Amélie Frégeau (1) ,1,2 Alexis Cournoyer, ${ }^{1,2}$ Marc-André Maheu-Cadotte (1) ,3,4 \\ Massimiliano Iseppon, ${ }^{5}$ Nathalie Soucy, ${ }^{6}$ Julie St-Cyr Bourque, ${ }^{7}$ Sylvie Cossette, ${ }^{8}$ \\ Véronique Castonguay, ${ }^{1,2}$ Richard Fleet $^{9}$
}

To cite: Frégeau A, Cournoyer $A$, Maheu-Cadotte M-A, et al. Use of tabletop exercises for healthcare education: a scoping review protocol. BMJ Open 2020;10:e032662. doi:10.1136/ bmjopen-2019-032662

- Prepublication history for this paper is available online. To view these files, please visit the journal online (http://dx.doi org/10.1136/bmjopen-2019032662).

Received 30 June 2019 Revised 12 December 2019 Accepted 13 December 2019

Check for updates

(c) Author(s) (or their employer(s)) 2020. Re-use permitted under CC BY-NC. No commercial re-use. See rights and permissions. Published by BMJ.

For numbered affiliations see end of article.

Correspondence to

Dr Amélie Frégeau;

amelie.fregeau@umontreal.ca

\section{ABSTRACT}

Introduction There is a growing interest in developing interprofessional education (IPE) in the community of healthcare educators. Tabletop exercises (TTX) have been proposed as a mean to cultivate collaborative practice. A TTX simulates an emergent situation in an informal environment. Healthcare professionals need to take charge of this situation as a team through a discussion-based approach. As TTX are gaining in popularity, performing a review about their uses could guide educators and researchers. The aim of this scoping review is to map the uses of TTX in healthcare.

Methods and analysis A search of the literature will be conducted using medical subject heading terms and keywords in PubMed, Medline, EBM Reviews (EvidenceBased Medicine Reviews), CINAHL (Cumulative Index of Nursing and Allied Health Literature), Embase and ERIC (Education Resources Information Center), along with a search of the grey literature. The search will be performed after the publication of this protocol (estimated to be January 1st 2020) and will be repeated 1 month prior to the submission for publication of the final review (estimated to be June 1st 2020). Studies reporting on TTX in healthcare and published in English or French will be included. Two reviewers will screen the articles and extract the data. The quality of the included articles will be assessed by two reviewers. To better map their uses, the varying TTX activities will be classified as performed in the context of disaster health or not, for IPE or not and using a board game or not. Moreover, following the same mapping objective, outcomes of TTX will be reported according to the Kirkpatrick model of outcomes of educational programs.

Ethics and dissemination No institutional review board approval is required for this review. Results will be submitted for publication in a peer-reviewed journal. The findings of this review will inform future efforts to TTX into the training of healthcare professionals.

\section{INTRODUCTION}

Most serious errors in critical care occur because of poor communication or collaboration rather than individual mistakes. ${ }^{1}$ Therefore, interest in interprofessional education (IPE) as a means to cultivate collaborative practice continues to grow among healthcare educators worldwide. $^{2}{ }^{3}$ According to the Centre for the Advancement in

\section{Strengths and limitations of this study}

The results of this study will be reported using a strategy based on the Preferred Reporting Items for Systematic Review and Meta-Analyses extension for Scoping Reviews (PRISMA-ScR) Checklist and the scoping methodological framework developed by Arksey and O'Malley.

- In addition to the standard requirements for scoping reviews, a formal quality assessment of included studies will be conducted using a checklist developed by Hawker and colleagues, as well as the JBI Critical Appraisal Checklist for Text and Opinion Papers (Joanna Briggs Institute).

- The study selection, the evaluation of the quality of the retained articles and data extraction will be performed by two independent reviewers to minimise the risk of bias or errors.

- The outcomes of each included study will also be reported according to the Kirkpatrick model of outcomes of educational programs.

- The search strategy proposed here is broad, but one of its limitation is the exclusion of articles published in languages other than English or French.

Interprofessional Education, IPE occurs when 'two or more professions learn with, from and about each other to improve collaboration and the quality of care'. ${ }^{4}$ IPE aims to develop competencies of collaborative practice in healthcare professionals including role clarification, patient-centred care, teamwork, collaborative leadership, interprofessional communication and interpersonal conflict resolution. ${ }^{5}$ Various pedagogical designs of IPE activities, such as tabletop exercises (TTX), have been used by healthcare educators to develop collaborative practice in healthcare professionals. ${ }^{67}$

A TTX is a facilitated group discussion that simulates an emergency situation in an informal, stress-free environment, sometimes using a board game format, and aims to strengthen readiness to manage a health emergency. TTX have been mostly used in disaster health to evaluate an organisation's 
preparedness to face a disaster and to educate healthcare professionals on their roles during the response. ${ }^{8-13}$ By using TTX as a simulation tool, hospital administrators can assess whether professional roles and responsibilities throughout the response system are well understood and accomplished promptly. ${ }^{14}$ TTX have also been used, to a lesser extent, in non-disaster health. ${ }^{15} 16$

No review has yet described the different uses of TTX. Hence, as TTX are gaining in popularity, performing a review about their uses is indicated. The aim of this scoping review is to map the uses of TTX in healthcare. Given the broad scope of this study, a scoping review methodology seems the best option as it aims 'to examine the extent, range and nature of research activity. This type of rapid review might not describe research findings in any detail but is a useful way of mapping fields of study where it is difficult to visualise the range of material that might be available'. ${ }^{17}$ In this scoping review, the various contexts in which TTX were used will be classified as in the field of disaster or non-disaster health, as an IPE or non-IPE exercise and whether or not they use a board game format. Moreover, an effort will be made to classify the reported outcomes of each selected study. The findings of this review will guide future research in this area and inform healthcare educators and administrators who are considering or developing TTX in their institution.

\section{METHODS}

The protocol for this scoping review is based on Arksey and O'Malley's five-stage methodological framework statement and reported according to the Preferred Reporting Items for Systematic Review and Meta-Analyses extension for Scoping Reviews (PRISMA) Checklist. ${ }^{17-19}$ The development of this protocol started in June 2018 and publication of the review is estimated for June 1st 2020.

\section{Stage 1: identifying the research question}

This scoping review aims to report the uses of TTX in healthcare and their outcomes.

\section{Definitions}

TTX are usually defined as a form of discussion-based activity that is guided by a facilitator. They usually involve a dialogue on the steps to take in response to a hypothetical scenario. Since there are various TTX designs, the definition that will be used in this scoping review is the following:

A tabletop exercise is an exercise that uses a progressive simulated scenario to make participants consider the impact of a potential health emergency on existing plans, procedures and capacities. A TTX simulates an emergency situation in an informal, stress-free environment. The purpose of a TTX is to strengthen readiness to manage a health emergency, through facilitated group discussions. ${ }^{20}$
The definition that will be used for disaster health is the following:

Disaster medicine is defined as the study [of] prevention, preparedness, response and recovery from the health problems arising from disaster. ${ }^{21}$

Contexts not attributed to the definition of disaster health will be classified as 'non-disaster health'.

The definition that will be used for IPE is the following:

When two or more professions learn with, from and about each other to improve collaboration and the quality of care. ${ }^{4}$

Contexts not attributed to the definition of IPE will be classified as 'non-IPE'.

The definition that will be used for board game TTX is the following:

A board game TTX can be real or virtual. The board depicts a disaster scene or a healthcare setting. The participants move around symbol units (also called "movable markers") which represent healthcare workers, patients and available resources to accomplish their duties. ${ }^{12}$

Contexts not attributed to the definition of board game TTX will be classified as 'non-board game TTX'.

\section{Research question}

In addition to classifying contexts of TTX's uses as pertaining to the field of disaster or non-disaster health, as an IPE or non-IPE exercise and whether or not they use a board game format, we plan to report the outcomes of each included study according to the Kirkpatrick Model of outcomes of educational programs. ${ }^{22}$ This model determines aptitude from training and educational programs based on four levels of criteria: reaction, learning, behaviour and organisational results. Generally, building an educational intervention that addresses higher levels of the Kirkpatrick model and measures higher level outcomes is more complex than building one that only addresses the lower levels. ${ }^{22}$ Therefore, the following research questions will be addressed:

- How have TTX been used in healthcare? More specifically, have they been used in the field of disaster or non-disaster health, as an IPE or non-IPE exercise and did they use a board game format?

- What were the outcomes of the studies on TTX in healthcare according to the Kirkpatrick Model of outcomes of educational programs? Was a level of outcome more represented than another?

While the primary focus of this review is to sum up in which contexts TTX have been used in healthcare and their outcomes, we will also provide a narrative review of the included studies regarding their design, setting, participants and interventions. If ever some contexts of use of TTX not identified beforehand emerge during the review process, they will be added iteratively. 


\section{Stage 2: identifying relevant studies}

Eligible studies, abstracts and conference summaries will be identified through a comprehensive search of CINAHL, Embase, EBM Reviews, ERIC, Medline and PubMed, while the grey literature will be searched using various online platforms (eg, Google Scholar). For practical reasons, searches will be limited to articles in English and French. There will be no limit on publication date in order to generate as broad a picture as possible of educational interventions in healthcare using TTX.

The search strategy was collaboratively and iteratively developed with the assistance of a librarian. We were unable to identify a medical subject heading that was specific for TTX. Hence, we developed a search strategy for PubMed using keywords related to the following concepts: TTX, whiteboards with magnetic symbols, simulation, training, serious games, desktop and board game. Open and closed vocabulary were used to determine the best possible strategy. The search strategy was adapted to search the other online databases, and similar keywords were used to search the grey literature.

This search strategy was initially developed on July 24th 2018 and improved during the revision of this manuscript. We plan to perform the search as soon as the present manuscript is accepted for publication (estimated to be January 1st 2020) and we will repeat it 1 month prior to the submission for publication of the final review (estimated to be June 1st 2020) to ensure it is still up to date.

\section{Stage 3: study selection}

\section{Inclusion criteria}

The eligibility of articles will be assessed based on the following inclusion criteria:

- The population of interest: healthcare professionals, including students in a healthcare program.

- The settings: academic and clinical settings where healthcare is provided or taught (eg, university, hospital, clinics).

- The intervention: a TTX as a stand-alone intervention or as part of a multicomponent intervention (eg, combined with a workshop or a classroom-based learning activity).

- The outcome: at least one learning outcome from the Kirkpatrick Model (as previously described) is reported.

- Study design: all types of study designs (eg, qualitative designs, quantitative designs and mixed methods designs) and methodologies including commentaries, case studies, descriptions of pedagogical innovations, conference summaries and viewpoint articles.

\section{Exclusion criteria}

- Scoping review and systematic review articles will be excluded.

- Studies published in a language other than English or French will be excluded.

EndNote (V.X9, Clarivate Analytics) will be used to import, manage, categorise and upload all collected references during the screening and selection process. If we are unable to obtain the full text of an article, we will contact the corresponding author for the article in question; failure to respond will result in the exclusion of the study. The reference lists of all included studies will be screened to search for any additional relevant studies.

Two reviewers (AF, AC) will meet to discuss the criteria for inclusion and will then independently screen the titles and abstracts.

Following the initial selection process, the full-text articles that are potentially relevant will be screened for eligibility by two reviewers (AF, M-AM-C). Any disagreements regarding study inclusion will be resolved through consensus. If consensus cannot be reached, a third reviewer (AC) will be consulted to resolve the disagreement. Reasons for excluding references at the full-text assessment stage of the screening process will be documented and reported in a PRISMA flow diagram. ${ }^{19}$ The reviewers will meet again following full-text assessment to discuss any challenges and uncertainties related to study selection.

\section{Stage 4: charting the data}

Two reviewers (AF, M-AM-C) will independently perform data extraction from studies included in the review. The research team has adapted a standardised charting form that was inspired by a protocol published by Shen $e t a l^{23}$ which was based on a similar research question that was applied to a different subject. This form was judged by members of our research team to be easy to use and relevant to our aims. The data charting domains and subdomains are described in table 1 .

Reviewers will pilot the charting form on five studies to determine whether this approach to data extraction is consistent with the research question and study purpose. Any relevant data that are not captured during the initial data extraction phase will be added iteratively by adapting the chart. If there is unclear or missing data in an article, we will contact the corresponding author to obtain clarification or additional data.

Although an assessment of study quality is not mandatory for a scoping review, it is strongly recommended by Arksey and O'Malley's five-stage methodological framework statement. ${ }^{17}$ We will assess the quality of included studies to more precisely describe the current evidence on TTX in healthcare and to formulate future-oriented advice that addresses methodological gaps identified in the literature. For original studies, study quality will be independently assessed by two reviewers (AF, M-AM-C) according to a checklist developed Hawker et $a .^{24}$ This tool was chosen because it has been validated to systematically review disparate data, whether qualitative or quantitative. For editorials, opinion texts and comments, we will assess quality using the validated JBI Critical Appraisal Checklist for Text and Opinion Papers. $^{25}$ 


\begin{tabular}{|c|c|}
\hline $\begin{array}{l}\text { Domain/ } \\
\text { subdomains }\end{array}$ & Description \\
\hline \multicolumn{2}{|l|}{ Article details } \\
\hline Author & Last name and initials of the first author \\
\hline Year & Publication year of the article \\
\hline Country & Country where the study was performed \\
\hline \multicolumn{2}{|l|}{ Initiative details } \\
\hline Context & $\begin{array}{l}\text { What was the need to organise a TTX? Is it } \\
\text { in the context of disaster health or of non- } \\
\text { disaster health? Is it in the context of IPE } \\
\text { or non-IPE? Were they using a board game } \\
\text { format or not? }\end{array}$ \\
\hline Setting & $\begin{array}{l}\text { Where did the educational intervention take } \\
\text { place (eg, community, hospital, university)? }\end{array}$ \\
\hline $\begin{array}{l}\text { Program } \\
\text { delivery }\end{array}$ & $\begin{array}{l}\text { How was the program delivered (eg, } \\
\text { seminar, lecture, course, in-service } \\
\text { training)? }\end{array}$ \\
\hline Instructors & Who were the facilitators/instructors? \\
\hline $\begin{array}{l}\text { Program } \\
\text { length }\end{array}$ & $\begin{array}{l}\text { How long did the program/intervention } \\
\text { last? }\end{array}$ \\
\hline \multicolumn{2}{|l|}{ Study details } \\
\hline Study design & What was the study design? \\
\hline Participants & $\begin{array}{l}\text { Who were the study participants? What } \\
\text { was the sample size? }\end{array}$ \\
\hline Intervention & What was the intervention? \\
\hline Comparator & What was the comparator? (if applicable) \\
\hline $\begin{array}{l}\text { Study } \\
\text { outcomes }\end{array}$ & $\begin{array}{l}\text { What did the authors identify as the study } \\
\text { outcomes? }\end{array}$ \\
\hline
\end{tabular}

$\begin{array}{cl}\text { Kirkpatrick's level } & \\ \text { Reaction } & \text { Did the intervention measure the immediate } \\ \text { perception and attitude of the learner } \\ \text { regarding the intervention? }\end{array}$

$\begin{array}{ll}\text { Learning } & \begin{array}{l}\text { Did the intervention measure what was } \\ \text { learnt during the pedagogical intervention? }\end{array} \\ \text { Behaviour } & \begin{array}{l}\text { Did the intervention measure if the learner } \\ \text { applied the new knowledge in their daily } \\ \text { life? }\end{array}\end{array}$

$\begin{array}{ll}\text { Results } & \text { Did the intervention measure the } \\ \text { organisational impact of daily use of the } \\ \text { new knowledge at work by the learner? }\end{array}$

Risk of bias

Hawker What is the score of the study? (if original checklist study)

$\mathrm{JBI}$ checklist What is the score of the study? (if editorial, opinion or comment)

IPE, interprofessional education; TTX, tabletop exercises.

Stage 5: Collating, summarising and reporting the results We will report data for each selected study including lead author, publication year, country, study context, setting, design, program delivery, program duration, participants, instructors, intervention, comparator (if applicable) and the outcomes. An effort will be made to report contexts of TTX according to three different characteristics:

I. Disaster health or non-disaster health.

II. IPE or non-IPE.

III. Board game or non-board game format.

This classification will facilitate the mapping of the current uses of TTX.

Moreover, we will classify the outcomes using the Kirkpatrick model of outcomes of educational programs for each study. ${ }^{22}$ We will report on all levels of Kirkpatrick's model, with special inquiry into the level that is most frequently represented among the studies included in the review.

Finally, results of the quality assessment will be reported using a checklist developed by Hawker et al for each original study, and using the JBI Critical Appraisal Checklist for Text and Opinion Papers for any editorials, opinion texts or comments included in the review. ${ }^{24} 25$

\section{Patient and public involvement}

No patients involved.

\section{ETHICS AND DISSEMINATION}

As this will be a scoping review of previously published studies, no ethics approval is required. The study findings will be submitted to an appropriate peer-reviewed journal.

\section{DISCUSSION}

This scoping review will fill an important gap in the literature, as there are no existing reviews that exclusively focusses on mapping the use of TTX in healthcare settings. The results of this study will inform researchers, healthcare educators, clinicians and administrators on the various uses of TTX in healthcare and more specifically in which contexts they are being used. Moreover, it will be possible to observe if the available literature focuses primarily on a single level of Kirkpatrick's model of outcome or ignores a particular level of outcome. These findings will be used to help develop and implement future educational programs involving TTX, with the hope that enhanced training of healthcare professionals will ultimately lead to improvements in patient, care, safety and satisfaction.

A limitation of this review is the exclusion of articles published in languages other than English or French.

\section{Author affiliations}

1Department of Emergency Medicine, Hôpital du Sacré-Coeur de Montréal, Montreal, Quebec, Canada

${ }^{2}$ Faculty of Medicine, Université de Montréal, Montreal, Quebec, Canada ${ }^{3}$ Research department, Institut de Cardiologie de Montréal, Montreal, Quebec, Canada

${ }^{4}$ Faculty of Nursing, Université de Montréal, Montreal, Quebec, Canada ${ }^{5}$ Department of Emergency Medicine, Hôpital Maisonneuve-Rosemont, Montreal, Quebec, Canada

${ }^{6}$ Direction of Education and of CHUM Academy, Centre hospitalier de I'Université de Montréal, Montreal, Quebec, Canada 
${ }^{7}$ Emergency Medicine Department, Centre hospitalier de I'Université de Montréal, Montreal, Quebec, Canada

${ }^{8}$ Research and International Development, Institut de Cardiologie de Montréal, Montreal, Quebec, Canada

${ }^{9}$ Department of Emergency Medicine, Université Laval, Lévis, Quebec, Canada

Acknowledgements This research will be carried out as part of the of the first author's Masters thesis, for which she received scholarships from the following: Centre de recherche sur les soins et les services de première ligne de l'Université Laval from Université Laval. The authors would like to thank Daniela Ziegler for helping with the design of the research methodology and Percipient Research \& Consulting for their assistance with language editing.

Contributors All authors contributed to the conception of the protocol. AF wrote the protocol. AC, M-AM-C, MI, NS, JS-CB, SC, VC and RF critically revised the draft for important intellectual content. All authors have approved the final version of this submitted manuscript and agree to be accountable for all aspects related to this work.

Funding This work was supported by a CAD $\$ 2500$ grant for research in primary care from Chaire du Docteur Sadok Besrour de l'Université de Montréal and a CAD\$5000 grant for research in primary care from Centre de recherche sur les soins et les services de première ligne de l'Université Laval and the Centre universitaire intégré de soins et de services de première ligne de la Capitale-Nationale.

Competing interests None declared.

Patient consent for publication Not required.

Provenance and peer review Not commissioned; externally peer reviewed.

Open access This is an open access article distributed in accordance with the Creative Commons Attribution Non Commercial (CC BY-NC 4.0) license, which permits others to distribute, remix, adapt, build upon this work non-commercially, and license their derivative works on different terms, provided the original work is properly cited, appropriate credit is given, any changes made indicated, and the use is non-commercial. See: http://creativecommons.org/licenses/by-nc/4.0/.

\section{ORCID iDs}

Amélie Frégeau http://orcid.org/0000-0002-7135-1145

Marc-André Maheu-Cadotte http://orcid.org/0000-0003-3190-0901

\section{REFERENCES}

1 Lehmann R, Seitz A, Meyburg J, et al. CAEP pediatric in-hospital emergencies: real life experiences, previous training and the need for training among physicians and nurses. BMC Research Note 2019;12:1-7.

2 Lutfiyya MN, Chang LF, McGrath C, et al. The state of the science of interprofessional collaborative practice: a scoping review of the patient health-related outcomes based literature published between 2010 and 2018. PLoS One 2019;14:1-18.

3 Baird J, Ashland M, Rosenbluth G. Interprofessional teams: current trends and future directions. Pediatr Clin North Am 2019;66:739-50.

4 Gum LF, Sweet L, Greenhill J, et al. Exploring interprofessional education and collaborative practice in Australian rural health services. J Interprof Care 2019;10:1-11.
5 Goldman J, Kitto S, Reeves S. Examining the implementation of collaborative competencies in a critical care setting: key challenges for enacting competency-based education. J Interprof Care 2018;32:407-15.

6 Danielson J, Willgerodt M. Building a theoretically Grounded curricular framework for successful interprofessional education. Am J Pharm Educ 2018;82:7075.

7 Wong A, Ruppel H, Gang M, et al. "The Safety Dance": A Faculty Development Workshop Partnering IPE and Patient Safety Initiatives Using Simulation-Based Education. MEP 2016;12:1-6.

8 Linskens EJ, Neu AE, Walz EJ, et al. Preparing for a foreign animal disease outbreak using a novel tabletop exercise. Prehosp Disaster Med 2018;33:640-6.

9 Chung S, Gardner AH, Schonfeld DJ, et al. Addressing children's needs in disasters: a regional pediatric tabletop exercise. Disaster Med Public Health Prep 2018;12:582-6.

10 Wexler B, Flamm A. Lessons learned from an active shooter full-scale functional exercise in a newly constructed emergency department. Disaster Med Public Health Prep 2017;11:522-5.

11 Savoia E, Biddinger PD, Fox P, et al. Impact of tabletop exercises on participants' knowledge of and confidence in legal authorities for infectious disease emergencies. Disaster Med Public Health Prep 2009;3:104-10.

12 Evans CA, Schwartz R. Using tabletop exercises as an innovative and practical teaching strategy in response to external disaster scenarios. Nurs Educ Perspect 2019;40:62-4.

13 Skryabina E, Reedy G, Amlôt R, et al. What is the value of health emergency preparedness exercises? A scoping review study. Int J Disaster Risk Reduct 2017;21:274-83.

14 Mackenzie C, Donohue J, Wasylina P, et al. How will military/civilian coordination work for reception of mass casualties from overseas? Prehosp Disaster Med 2009;24:380-8.

15 Lee Gordon D, Issenberg SB, Gordon MS, et al. Stroke training of prehospital providers: an example of simulation-enhanced blended learning and evaluation. Med Teach 2005;27:114-21.

16 Møller TP, Münter KH, Østergaard D, et al. Exploring challenges and solutions in the preparation of surgical patients. Dan Med J 2015;62:A5141.

17 Arksey H, O'Malley L. Scoping studies: towards a methodological framework. Int J Soc Res Methodol 2005;8:19-32.

18 Tricco AC, Lillie E, Zarin W, et al. PRISMA extension for scoping reviews (PRISMA-ScR): checklist and explanation. Ann Intern Med 2018;169:467-73.

19 Moher D, Liberati A, Tetzlaff J, et al. Preferred reporting items for systematic reviews and meta-analyses: the PRISMA statement. BMJ 2009;339:b2535.

20 World Health Organization. WHO simulation exercise manual. Geneva: World Health Organization, 2017.

21 A Bradt D, Abraham K, Franks R. A strategic plan for disaster medicine in Australasia. Emerg Med Australas 2003;15:271-82.

22 Kirkpatrick DL, Kirkpatrick JD. Evaluating training programs. 3rd edn. San Francisco, CA: Berrett-Koehler Publishers, 2006.

23 Shen N, Sockalingam S, Abi Jaoude A, et al. Scoping review protocol: education initiatives for medical psychiatry collaborative care. BMJ Open 2017;7:e015886.

24 Hawker S, Payne S, Kerr C, et al. Appraising the evidence: reviewing disparate data systematically. Qual Health Res 2002;12:1284-99.

25 Aromataris E, Munn Z. Joanna Briggs Institute Reviewer's Manual. The Joanna Briggs Institute, 2017. Available: https:// reviewersmanual.joannabriggs.org/ 